\section{POS0581 PERIODONTAL DISEASE IN RHEUMATOID ARTHRITIS: RESULTS FROM A COHORT AT TREATMENT WITH BIOLOGICAL, CLASSICAL AND TARGETED SYNTHETIC DMARDS}

R. Dos-Santos ${ }^{1}$, F. Otero ${ }^{1}$, E. Perez-Pampín ${ }^{1}$, A. Mera Varela ${ }^{1} .{ }^{1}$ Clinical University Hospital in Santiago de Compostela, Rheumatology Department, Universidad de Santiago de Compostela, Santiago de Compostela, Spain

Background: Periodontal disease (PD) has been widely studied in the pathogenesis of rheumatoid arthritis (RA). As well, its relationship with severity and disease activity, has also been investigated with ambiguous results. It has been suggested that the improvement of oral health could enhance disease activity scores. ${ }^{1}$ PD prevalence worldwide stands around $60 \%$ in older adults (>65 years) and its frequency increases with aging. ${ }^{2}$

Objectives: To asses oral health in RA patients and to identify predictors of PD in this population.

Methods: Patients diagnosed of RA at treatment with biological, classical or targeted synthetic disease modifying anti-rheumatic drugs (b/cs/tsDMARDs) in the aforementioned hospital during 2020 performed a dental review with a specialized periodontal odontologist. Oral health patterns were given for all patients, following criteria of American Academy of Periodontology, and reevaluation of disease activity was made 2 months later.

Clinical, demographic and treatment data were collected from participants. Univariable logistic regression was performed to identify predictors of PD. Variables with $p<0.20$ were selected for multivariable analysis.

Stata 15.1 was used to perform statistical analysis.

Results: 81 patients were recruited. $82.72 \%$ were female. Mean age was 56.17 years (SD 14.15) and mean time since diagnosis was 15.58 years (SD 8.17) $25 \%$ were current or past smokers. 21 patients had comorbidities (arterial hypertension the most frequent). $66.67 \%$ were rheumatoid factor (RF) positive and $72.73 \%$ anti-citrullinated peptide autoantibody (ACPA) positive. Median erythrocyte sedimentation rate (ESR) was $12 \mathrm{~mm}$ (IQR 6;23) and mean C-reactive protein (CRP) was $0.48 \mathrm{mg} / \mathrm{dl}$ (SD 1.18). Mean disease activity score (DAS28-VSG) at the testing time was 2.62 (SD 1.21) and after 2 months was 2.39 (SD 0.97) $96.30 \%$ of patients were at treatment with csDMARDs, $64.20 \%$ with glucocorticoids, $96.30 \%$ with bDMARDs and 6 patients with tsDMARDs.

Univariable analysis identified higher age, at least one autoantibody positive and $\mathrm{ESR} / \mathrm{CRP}$ as potential predictors of medium/severe PD $(p<0.20)$. Multivariable testing including these variables pointed out higher age, lower ESR and at least one autoantibody positive (OR 1.09 [Cl95\% 1.04-1.14] $\mathrm{p}=0.001$, OR 0.18 [Cl95\% $0.04-0.95$ ] $p=0.044$ and OR 0.94 [Cl95\% 0.88-1.00] $p=0.042$, respectively) as predictors of medium or severe PD ( $\geq 3 \mathrm{~mm}$ interdental clinical attachment loss). Univariable analysis identified higher age, the presence of any comorbidity and anti tumour-necrosis factor alpha treatment (anti-TNF) as potential predictors of severe PD $(p<0.20)$. Multivariable testing including these variables pointed out higher age (OR $1.15[\mathrm{Cl} 95 \% 1.02-1.30] \mathrm{p}=0.026$ ) as predictor of severe PD ( $\geq 5 \mathrm{~mm}$ interdental clinical attachment loss).

Conclusion: Periodontal disease is still an extended health problem among the entire population. Its prevalence in RA is increased, therefore higher age and RF or ACPA positive are risk factors for developing severe PD. This analysis might suggest that an aggressive management of PD could implement better responses in DAS28. Also anti-TNF treatment could delimit a "penumbra" group of patients at risk of developing severe PD, where intensive manage could modify the final outcome.

REFERENCES:

[1] C O Bingham, M Moni. Periodontal disease and rheumatoid arthritis: the evidence accumulates for complex pathobiologic interactions. Curr Opin Rheumatol. 2013;25(3):345-353.

[2] P Carvajal. Periodontal disease as a public health problem: the challenge for primary health care. Rev Clin Periodoncia inplantol. 2016;9(2):177-183.

Disclosure of Interests: None declared

DOI: 10.1136/annrheumdis-2021-eular.3970

\section{POS0582 RHEUMATOID ARTHRITIS AND ORAL MICROBIOME: PREDICTORS OF DISTURBANCES AND ITS IMPLICATIONS. RESULTS FROM A COHORT AT TREATMENT WITH cs/b/tsDMARD}

R. Dos-Santos ${ }^{1}$, F. Otero ${ }^{1}$, E. Perez-Pampín ${ }^{1}$, A. Mera Varela ${ }^{1}{ }^{1}$ Clinical University Hospital in Santiago de Compostela, Rheumatology Department, Universidad de Santiago de Compostela, Santiago de Compostela, Spain

Background: Oral microbiome (OM) seems to be significant in the pathogenesis of some immune-mediated diseases, such as rheumatoid arthritis (RA), psoriasis or inflammatory bowel disease. ${ }^{1}$ Some microorganisms, as Porphyromonas gingivalis have been related with the production of autoantibodies. Also it has been suggested that composition of OM could change RA disease course, being more difficult-to-treat and having higher disease activity scores. ${ }^{2}$
Objectives: To identify which variables could predict the appearance of altered $\mathrm{OM}$ and its implications in clinical practice.

Methods: Patients were recruited if they were diagnosed of RA and were at active treatment (biological, classical or targeted synthetic disease modifying anti-rheumatic drugs [b/cs/tsDMARDs]). Patients performed a dental review with a specialized odontologist that made an OM test (semiquantitative PCR), and oral health standards were instructed (following criteria of American Academy of Periodontology). Recruitment was made during 2020 in the Clinical University Hospital in Santiago de Compostela, Spain. Disease activity reevaluation was made 2 months later.

Treatment, demographic and clinical data were collected from participants. Univariable logistic and linear regression were performed to identify predictors of $\mathrm{OM}$. Variables with $\mathrm{p}<0.20$ were selected for multivariable analysis.

Stata 15.1 was used to perform statistical analysis.

Results: 47 patients were selected of whom 40 were female. Mean age was 55.43 years (SD 14.42). $30.77 \%$ were current or ex-smokers. Mean time since RA diagnosis was 14.89 years (SD 8.47). $63.83 \%$ were anti-citrullinated peptide autoantibody (ACPA) positive and $70.21 \%$ were rheumatoid factor (RF) positive, letting only 6 patients double negative. $46.81 \%$ had moderate/severe periodontal disease (PD). $32.61 \%$ of patients had any comorbidity. Mean DAS28 at the OM test was 2.67 (SD 1.28) and after 2 months 2.37 (SD 1.03). Mean C-reactive protein (CRP) was $0.64 \mathrm{mg} / \mathrm{dl}$ (SD 1.48) and median erythrocyte sedimentation rate (ESR) was $13 \mathrm{~mm}$ (IQR 7;27). All patients were under glucocorticoid treatment, 46 with bDMARD, 1 with tsDMARD and 46 with csDMARD. Treponema denticola was detected in $44.68 \%$ of patients, P. gingivalis in $29.79 \%$, Actinomyces spp in $8.51 \%$, Tanerella forsythia in $36.17 \%$ and Prevotella intermedia in $25.53 \%$. Only 15 patients were full-negative for OM test.

Univariable analysis identified RF positive, double autoantibody positive (RF and ACPA) and moderate/severe PD as potential predictors of the presence of at least one oral microorganism $(\mathrm{p}<0.20)$. Multivariable testing pointed out moderate/severe PD as predictor of the presence of at least one oral microorganism (OR 22.91 [Cl95\% 2.38-220.4] p=0.007).

Univariable analysis identified higher age, presence of any comorbidity, RF positive, higher CRP, treatment with anti-tumour necrosis alpha (aTNF) and moderate/severe PD as potential predictors of the presence of multiple species in OM $(p<0.20)$. Multivariable testing pointed out moderate/severe PD as predicto of the presence of multiple species in OM (B $0.39[95 \% \mathrm{Cl} 0.19-0.58] \mathrm{p}=0.000)$. Conclusion: Oral microbiome is closely related with periodontal disease, added to our results, a relationship between $\mathrm{OM}$ and disease activity has been exposed. In this analysis the role of OM and autoantibody profile is manifest, as being double positive or RF positive is associated with the presence of altered $\mathrm{OM}$. Also patients with high acute-phase reactants, active disease and under aTNF treatment could delineate a specific RA population under risk of altered $\mathrm{OM}$, where intensive strategies for changing oral microbiome could have any repercussion in the disease course.

\section{REFERENCES:}

[1] Chen, B., Zhao, Y., Li, S. et al. Variations in oral microbiome profiles in rheumatoid arthritis and osteoarthritis with potential biomarkers for arthritis screening. Sci Rep 8, 17126 (2018).

[2] R Bodkhe, B Balakrishnan, V Taneja. The role of microbiome in rheumatoid arthritis treatment. Ther Adv Musculoskelet Dis. 2019;11:1759720.

Disclosure of Interests: None declared

DOI: 10.1136/annrheumdis-2021-eular.3979

\section{POS0583 ENGINEERED GLOVE TO EVALUATE THE SPEED
OF THE HANDS' MOVEMENTS IN RHEUMATOID
ARTHRITIS}

M. Patanè ${ }^{1}$, L. Carmisciano ${ }^{2}$, E. Hysa ${ }^{3}$, E. Gotelli ${ }^{3}$, A. Signori ${ }^{2}$, V. Smith ${ }^{4}$ M. Cutolo ${ }^{3} .{ }^{1}$ Ospedale Alessandria, Internal Medicine, Alessandria, Italy; ${ }^{2}$ University of Genoa, Department of Health Sciences, Genoa, Italy; ${ }^{3}$ University of Genoa, Research Laboratory and Academic Unit of Clinical Rheumatology, Department of Internal Medicine, IRCCS "San Martino" Polyclinic Hospital, Genova, Italy; ${ }^{4}$ Ghent University, Department of Internal Medicine; Department of Rheumatology; Unit for Molecular Immunology and Inflammation, Ghent University Hospital, Gent, Belgium

Background: Rheumatoid arthritis (RA) is a long-term, progressive, and disabling autoimmune disease1. The disease activity can be quantified by the Disease Activity Score 28-joint count - C reactive protein (DAS28crp)2; the evaluation of disability function (DF) is actually mainly performed only by subjective Patient Reported Outcomes (PROs) like Health Assessment Questionnaire (HAQ)3; to investigate the functional aspects of RA hands it is usually used the grip strength (GS)4. However, in the scientific literature no tool, which objectively evaluates movement speed, has been reported. The Hand Test System (HTS ETT) is an engineered glove (RAGLOVE), nowadays applied for neuroscience studies to evaluate hand motility5 
Objectives: To objectively evaluate the RA hand's speed of the fine movements, through the HTS and to compared with a group of age and sex matched healthy controls. To verify the correspondence with the HAQ, DAS28, GS

Methods: 55 consecutives RA patients (pts) ( 6 males, age $61 \pm 16$ years, mean duration of disease $12 \pm 8$ years), classified according to 2010 ACR/EULAR criteria6, and 50 matched healthy controls (HCs) were enrolled. After consent, all participants undergone HTS test that recognizes the touches between the finger tips during the opposition movements of the hands in standard sequences of movements, after dressed the glove. A multiple finger evaluation (MFE) and a single finger evaluation (SFE) were performed using a dedicated software that provided the physician the following quantitative parameters: Touch Duration (TD), Inter Tapping Interval (ITI) and Movement Rate (MR). Average time for hand 2 minutes. RA pts compiled the HAQ, performed the GS and a DAS28cpr was performed.

The student's t-test was used to compare the glove's parameters between the groups whereas the analysis of variance (ANOVA) was utilized to verify potential differences between the populations. In order to evaluate the single correlations, the $r$ and $p$ values of Pearson were employed.

Results: For MFE, glove parameters TD and ITI were significantly higher in RA pts than HCs, whereas; MR was significantly lower in RA pts compared to HCs (all $p<0.001$ ).

For SFE non-affected fingers (not swollen and not tender) of RA pts performed better than a clinically affected fingers, but in any case significantly worse than average HCs fingers $(p<0.001)$.

There is a statistically significant correlation between the GS and MR $(r=0.39$ $\mathrm{p}=0.003)$ and TD $(\mathrm{r}=-0.33 \mathrm{p}=0.015)$.

TD, ITI e MR of RA pts showed a significant correlation with the total score of the HAQ ( $r=0.56, r=0.39, r=-0.56$, all $p<0.001 ;)$. DAS28, considered as a continuous variable, proved to be significantly correlated with the TD $(r=0.36, p$ $=0.009)$. When the RA patients were grouped according to the disease activity by DAS28cpr7, there was an increase of one third of the TD's logarithm for each increase in the activity class (linear regression with ordinal predictors, beta = $0.33 ; 95 \% \mathrm{Cl} 0.03,0.63, \mathrm{p}<0.0297)$. Finally, even RA pts in remission showed a TD significantly higher compared with $\mathrm{HCs}(p=0.034)$.

Conclusion: The RAGLOVE is shown as a new safe and fast tool to evaluate a new objective parameter in the hand's functionality: the speed of finger movements. In RA pts, an inversely proportional correlation emerges between the speed of movement and disease activity.

The significant correlation found with $\mathrm{HAQ}$, highlights the loss of motility of the hands as one of the main determinant of disability. The RAGLOVE is now tested in RA patients undergoing treatment.

REFERENCES:

[1] Hakkinen et al Ann Rheum Dis. 2005;

[2] Van Der Heijde et al J of Rheum. 1993;

[3] Fries et al Arthritis Rheum. 1980;

[4] Mathiowetz et al J Hand Surg Am. 1984;

[5] Carmisciano et al Eur J Neurol. 2020;

[6] Aletaha et al. Ann Rheum Dis. 2010;

[7] Aletaha et al Arthritis Rheum 2005.

Disclosure of Interests: None declared

DOI: 10.1136/annrheumdis-2021-eular.3991

\section{POS0584 ULTRASONOGRAPHY OF THE MEDIAN NERVE IN PATIENTS WITH RHEUMATOID ARTHRITIS UNDER SUSPICION OF CARPAL TUNNEL SYNDROME}

S. Tsiami ${ }^{1}$, E. Ntasiou ${ }^{2}$, C. Krogias ${ }^{2}$, R. Gold ${ }^{2}$, J. Braun ${ }^{1}$, M. Sarholz ${ }^{3}$, X. Baraliakos ${ }^{1} .{ }^{1}$ Ruhr-University Bochum, Rheumazentrum Ruhrgebiet, Herne, Germany; ${ }^{2}$ Ruhr-University Bochum, St. Josef-Hospital, Bochum, Germany; ${ }^{3}$ St. Marien-Hospital Vreden, Klinik fuer Rheumatologie, Vreden, Germany

Background: Carpal tunnel syndrome (CTS) is the most common nerve compression syndrome and a common extra-articular manifestation of rheumatoid arthritis (RA). Different causes of CTS are known, among them inflammatory and non-inflammatory pathologies. Electroneurography (ENG) of the median nerve, the method of choice to diagnose CTS, measures impairment of nerve conduction velocity without explaining its underlying cause. However, because the electrical stimulation is often not well tolerated, ENG results may come out inconclusive. Using greyscale ultrasonography (GS-US) provides anatomic information including a structural representation of the carpal tunnel.

Objectives: To investigate the performance of nerve GS-US in the diagnosis of CTS in patients with RA.

Methods: Consecutive patients with active RA under suspicion of CTS presenting to a large rheumatologic center were included. Both hands were examined by an experienced neurologist including ENG and a GS-US (ML linear probe with 6-15 Hz) of the median nerve. An established grading system for ENG (1), and an established system for GS-US based on cut-offs for the nerve cross sectional area (CSA) [mild: $0,11-0,13 \mathrm{~cm}^{2}$, moderate: $0,14-0,15 \mathrm{~cm}^{2}$, severe: $>0,15 \mathrm{~cm}^{2}$ CTS
(2)] were used. In addition, the Boston Carpal Tunnel Syndrome Questionnaire (BCTSQ) was used to assess CTS symptoms (3).

Results: Both hands of 58 patients with active RA $(n=116)$ and clinical suspicion of CTS (in 38 cases bilaterally) were included. After clinical examination, CTS was suspicious in 96 hands (82.8\%), and 59 of all hands had a final diagnosis of CTS $(50.9 \%)$. Of the latter, 43 hands $(72.9 \%)$ had a positive ENG and 16 $(27.1 \%)$ a positive GS-US finding only, while 30 hands $(50.8 \%)$ were positive in both examinations.

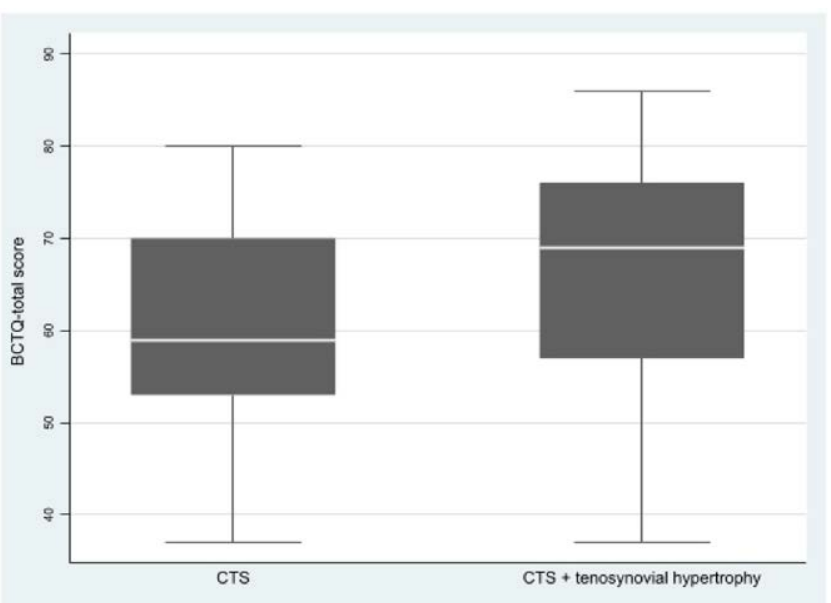

Figure 1. BCTSQ scores in patients with diagnosis of CTS and absence or presence of RA-related tenosynovial hypertrophy

There was a good correlation of the cross-sectional area (CSA) as well as the CSA-ratio to the ENG findings: the larger the CSA, the more severe was the CTS as assessed by ENG (Spearman's rho $=0.554$; $p<0.001$ ). The more severe the GS-US findings of CTS were, the more definite were the distal motor latency (Spearman's rho $=0.554 ; p<0.001$ ) and sensible nerve conduction velocity of the median nerve (Spearman's rho $=-0.5411 ; p<0.001$ ).

In the 46 hands positive in GS-US, tenosynovial hypertrophy of the flexor tendons was detected in 19 hands (41.3\%), 7 of which (36.8\%) also showed an additional cystic mass. In these 19 patients, clinical complains were more severely present than in patients with non-inflammatory CTS, as assessed by the BCTSQ with a total score of $68.8 \pm 13.4$ vs. $59.3 \pm 13.7$, respectively $(p=0.007)$.

Conclusion: In patients with active RA and clinical complains of CTS, ultrasound examinations provide additional information about inflammation which is helpful for a diagnosis of CTS. Thus, ENG and nerve GS-US should be used complementary for a diagnostic workup of CTS in RA patients with a suspicion of CTS. Power-Doppler may further improve the diagnostic performance of GS-US. REFERENCES:

[1] Padua L et al. Acta Neurol Scand 1997; 96:211-217

[2] El Miedany et al., Rheumatology (Oxford). 2004 Jul; 43(7):887-895

[3] Levine DW et al. J Bone Joint Surg Am 1993; 75: 1585-1592

Disclosure of Interests: None declared

DOI: 10.1136/annrheumdis-2021-eular.3994

\section{ORAL STATUS IN PATIENTS WITH RHEUMATOID ARTHRITIS}

A. Ben Salem ${ }^{1}$, M. Brahem ${ }^{1}$, H. Hachfi ${ }^{1}$, R. Sarraj ${ }^{1}$, S. Abdellatif ${ }^{1}$, R. Ben Tekaya ${ }^{1}$, C. Baccouche ${ }^{2}$, Y. Mohamed ${ }^{1} .{ }^{1}$ Tahar Sfar Hospital, Rheumatology, Mahdia, Tunisia; ${ }^{2}$ Tahar Sfar Hospital, Dentistry, Mahdia, Tunisia

Background: Rheumatoid arthritis (RA) is a systemic auto immune inflammatory disorder, which primarily affects the synovial joints, but it may have an impact on oral health.

Objectives: The present study aimed to describe oral manifestations in patients with Rheumatoid arthritis (RA)

Methods: A cross sectional study including RApatients, which consulted or were hospitalized in Rheumatology department in Taher Sfar Hospital of Mahdia Tunisia, during a period of 10 months. The diagnosis of RA was based on ACR/EULAR 2010 criteria. Oral and periodontal examination was practiced by a trained dentist in the same hospital. Oral hygiene, teeth status (missing teeth) and paradental parameters (bleeding index of Loe and Silness (IG), plaque indexed O'Leary (IP) were determined for each patient, to assess oral and periodontal diseases in RA.

Results: Our study included 51 patients with an average age of 51.11 years \pm 12.4 [21-74years].50patients (92.6\%of cases) were women and 8patients (14.8\%) were diabetic. Only one patient was a smoker. The mean duration of RA was 10.7 years $7.7 \pm$ [10months-35years]. Rheumatoid factor (RF) was positive 